\title{
Understandings of Classical and Incremental Backstepping Controllers with Model Uncertainties
}

\author{
Byoung-Ju Jeon, Min-Guk Seo, Hyo-Sang Shin, and Antonios Tsourdos
}

\begin{abstract}
This paper suggests closed-loop analysis results for both classical and incremental backstepping controllers considering model uncertainties. First, transfer functions with each control algorithm under the model uncertainties, are compared with the ones for the nominal case. The effects of the model uncertainties on the closed-loop systems are critically assessed via investigations on stability conditions and performance metrics. Second, closed-loop characteristics with classical and incremental backstepping controllers under the model uncertainties are directly compared using derived common metrics from their transfer functions. This comparative study clarifies how the effects of the model uncertainties to the closed-loop system become different depending on the applied control algorithm. It also enables understandings about the effects of additional measurements in the incremental algorithm. Third, case studies are conducted assuming that the uncertainty exists only in one aerodynamic derivative estimate while the other estimates have true values. This facilitates systematic interpretations on the impacts of the uncertainty on the specific aerodynamic derivative estimate to the closed-loop system.
\end{abstract}

Index Terms-Backstepping Control, Incremental Backstepping Control, Model Uncertainty, Closed-loop Analysis, Model based Algorithm, Sensor based Algorithm

\section{INTRODUCTION}

B ACKSTEPPING algorithm [1] is one of the most widely and successfully applied nonlinear flight control methods [2]-[11]. The backstepping(BKS) controller design starts from dynamics farthest from a control input and then steps back through integrators by considering augmented Lyapunov functions, to obtain the controller which fulfils desired motions with known stability and convergence properties. Since BKS requires explicit model information to implement the algorithm, it can be regarded as a model based approach. The issue is that a model based control strategy is normally sensitive to model uncertainties, but it could be hard to obtain accurate model information.

Incremental backstepping(IBKS) method [12]-[15] is suggested to reduce model dependency of BKS. This incremental controller utilizes additional measurements such as state derivatives and control surface deflection angles, replacing a part of required model information. This algorithm becomes implicit, not totally residing in explicit model to be cancelled. Because IBKS still requires knowledge about control effectiveness in its implementation, it lies in between model based and sensor based approach.

B.-J. Jeon, M.-G. Seo, H.-S. Shin and A. Tsourdos are with School of Aerospace, Transport and Manufacturing (SATM), Cranfield University, College Road, Cranfield, United Kingdom (e-mail : h.shin@cranfield.ac.uk)

Manuscript received October 25, 2018
Not only for BKS but also for IBKS, it is essential to identify and understand the effects of the model uncertainties on each closed-loop system. There have been some studies [12]-[15] that investigated their closed-loop characteristics under the model uncertainties, but only by numerical simulations or experiments. Since results obtained from simulations or experiments are valid for particular plants under specific conditions, theoretical analysis is necessary for general interpretations on the effects of the model uncertainties to the closed-loop systems with BKS and IBKS. To the best of our knowledge, it is difficult to find existing studies that successfully provide theoretical analysis on BKS and IBKS considering the model uncertainties. Hence, this paper aims to suggest the closed-loop analysis results on BKS and IBKS, especially in consideration of the model uncertainties.

Unlike BKS and IBKS, there has been several researches [16]-[20] in which closed-loop analysis under the model uncertainties was carried out for nonlinear dynamic inversion(NDI) and incremental nonlinear dynamic inversion(INDI). Note that NDI [21] is another representative nonlinear control approach, most widely developed and applied [22]-[25]. NDI explicitly cancels out undesired nonlinearities in system using inverse dynamics, and INDI is its incremental version, similar to IBKS. BKS and NDI have some similarities, since both nonlinear controllers ultimately try to make error dynamics of a closed-loop system to achieve desired behaviors by cancelling undesired nonlinearities in dynamics. Therefore, it would be worth to examine previous analyses on NDI / INDI and investigate possibility to extend them to the analysis of BKS / IBKS.

In [16], each of NDI and INDI was applied to an innerloop of 6-DoF nonlinear dynamics for an unmanned aerial vehicle. Under model uncertainties, a transfer function with INDI was suggested, but closed-loop analysis with NDI was difficult to proceed further due to remaining nonlinearities. For the closed-loop analysis with INDI, incremental dynamics utilized in control law derivation process was applied instead of true system dynamics. This makes difficult to comprehend the effects of the control surface deflection measurement to the system. Besides, it is hard to find comparative study between INDI and NDI in closed-loop characteristics under the model uncertainties. [18] performed analysis with NDI and INDI under a general type of nonlinear dynamics based on Lyapunov stability theory. Consequently, proof of bounded stability for the closed-loop systems and conditions to achieve it were provided. This Lyapunov-based analysis was mathematically rigorous, but it had limitations to get physical understandings for applications on real systems. Additionally, analysis consid- 
ering the model uncertainties was conducted, addressing rough understandings about their effects. It is difficult to investigate detailed effects of the model uncertainties on stability and performance.

The review on analysis of NDI and INDI suggests that they could shed some lights on potential direction of our analysis. However, they cannot be direct references for our research with BKS and IBKS, due to the following difference between BKS and NDI. NDI disregards interconnections between the loops under the time-scale separation assumption. On the contrary, BKS intermediately adopts the time-scale separation assumption, but it considers the transient responses in the end. This difference can make the analysis with BKS and IBKS more challenging than the one with NDI and INDI.

To this end, this paper suggests theoretical closed-loop analysis with BKS and IBKS under the model uncertainties. This analysis enables critical understandings on system characteristics related to the model uncertainties. Note that short period mode dynamics for an aircraft is utilized for simplicity of the analysis. BKS and IBKS are designed to achieve asymptotic stability of the system for the nominal case. Considering the model uncertainties, closed-loop analysis is conducted with a piece-wise approach. Transfer functions with BKS and IBKS under the model uncertainties, are compared with the ones for the nominal case. This shows important changes on the transfer functions due to the model uncertainties, resulting in conditions to maintain stability and performance metrics under the model uncertainties. By investigating distinctions between the transfer functions with two algorithms, it is clarified how the effect of the model uncertainties to the closed-loop system becomes different depending on the applied control algorithm. This comparative study also enriches understandings about the effect of additional measurements in IBKS. To have more insights from simplified situations, case studies are conducted under the assumption that the uncertainty exists only in one aerodynamic derivative estimate while the other estimates have true values. This facilitates systematic interpretations on the impacts of the uncertainty on the specific aerodynamic derivative estimate to the closed-loop system. Simulation is performed to verify properties obtained from the analysis.

\section{PReliminaries : DYNAMiCS}

From Newton's law of motion about conservation of linear and angular momentum, 6-DoF nonlinear coupled dynamics for an aircraft can be derived. Taylor series expansion provides the first order approximation in the neighborhood of trim points. Then, longitudinal and lateral motions can be decoupled under several flight conditions like a level flight. Short period mode is one of the longitudinal oscillation modes with high natural frequency. This mode is of paramount importance in flight control, because one of the main purposes of a stability augmentation system for an aircraft is to improve short period mode characteristics. Since the oscillation lasts for relatively short time, velocity change is assumed to be negligible. This results in the dynamics (1) below [26], and in this paper, it is applied for control law derivation and closedloop analysis.

$$
\begin{aligned}
& \dot{\alpha}=Z_{\alpha}^{*}(M, \alpha) \alpha+q+Z_{\delta}^{*}(M, \alpha) \delta \\
& \dot{q}=M_{\alpha}^{*}(M, \alpha) \alpha+M_{q}^{*}(M, \alpha) q+M_{\delta}^{*}(M, \alpha) \delta
\end{aligned}
$$

where

$$
\begin{aligned}
Z_{\alpha}^{*}(M, \alpha)= & \frac{\bar{q} S}{m} C_{Z_{\alpha}}(M, \alpha) \frac{1}{U_{0}} \\
Z_{\delta}^{*}(M, \alpha)= & \frac{\bar{q} S}{m} C_{Z_{\delta}}(M, \alpha) \frac{1}{U_{0}} \\
M_{\alpha}^{*}(M, \alpha)= & \frac{\bar{q} S \bar{c}}{I_{y}} C_{M_{\alpha}}(M, \alpha) \\
& +\frac{\bar{q} S \bar{c}^{2}}{2 I_{y} U_{0}} C_{M_{\dot{\alpha}}}(M, \alpha) \frac{\bar{q} S}{m} C_{Z_{\alpha}}(M, \alpha) \frac{1}{U_{0}} \\
M_{q}^{*}(M, \alpha)= & \frac{\bar{q} S \bar{c}^{2}}{2 I_{y} U_{0}} C_{M_{q}}(M, \alpha)+\frac{\bar{q} S \bar{c}^{2}}{2 I_{y} U_{0}} C_{M_{\dot{\alpha}}}(M, \alpha) \\
M_{\delta}^{*}(M, \alpha)= & \frac{\bar{q} S \bar{c}}{I_{y}} C_{M_{\delta}}(M, \alpha) \\
& +\frac{\bar{q} S \bar{c}^{2}}{2 I_{y} U_{0}^{2}} C_{M_{\dot{\alpha}}}(M, \alpha) \frac{\bar{q} S}{m} C_{Z_{\delta}}(M, \alpha)
\end{aligned}
$$

State variables $\alpha$ and $q$ represent an angle of attack and a pitch rate. Control input $\delta$ corresponds to a deflection of an elevator. $\bar{q}$ indicates a dynamic pressure, $U_{0}$ means a constant velocity of an aircraft, and $M$ stands for Mach number. For notational convenience, aerodynamic derivatives will be expressed in shorthand form as $Z_{\alpha}^{*}, Z_{\delta}^{*}, M_{\alpha}^{*}, M_{q}^{*}$ and $M_{\delta}^{*}$, but they are still functions of $M$ and $\alpha . C_{(\cdot)}$ denotes dimensionless aerodynamic coefficients. $S, \bar{c}, m$ and $I_{y}$ are reference area, reference length, mass and moment of inertia in $y$-axis of an aircraft.

Dynamics (1) represents a linear parameter-varying(LPV) system, i.e., a nonlinear system which can be described as a parametrized linear system whose parameters change with the states. This simplified version of dynamics, not full 6DoF dynamics, is utilized for simplicity of the analysis, because complex dynamics can make analysis with model uncertainties more complicated. Since the main objective in this study is to have critical understandings about the closedloop characteristics with BKS and IBKS under the model uncertainties, dynamics (1) is reasonable for this purpose. As a future work, this research can be extended to the analysis with full 6-DoF dynamics.

\section{DERIVATION OF CONTROL LAWS}

Before derivation of BKS and IBKS, following modification and assumption widely accepted in controller design phase, are applied to dynamics (1).

First, aerodynamic derivatives estimates $(\hat{)})$ are utilized instead of real $Z_{\alpha}^{*}, Z_{\delta}^{*}, M_{\alpha}^{*}, M_{q}^{*}$ and $M_{\delta}^{*}$, as only estimated values are available in controller design phase. Those derivatives are calculated from the dimensionless aerodynamic coefficients $C_{(\cdot)}$ identified from wind tunnel test or aeroprediction, and the aircraft parameters $S, \bar{c}, m$ and $I_{y}$ measured before flight. Hence, the aerodynamic derivatives estimates $(\hat{.})$ are most likely to contain uncertainties which make them different with their true values. Nevertheless, in this section, both controllers are designed to accomplish asymptotic stability 
assuming that the estimates are the same as their true values. The effects of the model uncertainties, which can make aimed performance and stability characteristics in this design phase difficult to be achieved, will be investigated in the closed-loop analysis part IV.

Second, $\hat{Z}_{\delta}^{*} \delta$ related to non-minimum phase is neglected, to make the system in lower-triangular form. Both control laws are based on the backstepping method, which requires that the dynamics should be in strict feedback form. Therefore, it is assumed that a fin surface is a pure moment generator. This is a valid assumption for most of aircrafts, often made in flight control systems design, because $C_{Z_{\delta}}$ is usually small enough [26].

Under these modification and assumption, the dynamics (2) below is utilized for the control law derivations.

$$
\begin{aligned}
& \dot{\alpha}=\hat{Z}_{\alpha}^{*} \alpha+q \\
& \dot{q}=\hat{M}_{\alpha}^{*} \alpha+\hat{M}_{q}^{*} q+\hat{M}_{\delta}^{*} \delta
\end{aligned}
$$

State errors are defined as follows.

$$
\begin{aligned}
& z_{1}=\alpha-\alpha_{c} \\
& z_{2}=q-q_{c}
\end{aligned}
$$

where subscript $c$ represents a command.

\section{A. Backstepping Control}

If Lyapunov function candidate becomes positive definite and its derivative becomes negative definite, asymptotic stability can be guaranteed for the system. To derive BKS control command which satisfies asymptotic stability, 2 cascaded steps are performed as follows.

First, Lyapunov function candidate $V_{1}$ considering only $z_{1}$ is selected as

$$
V_{1}=\frac{1}{2} z_{1}^{2}
$$

which is positive definite. It's for the outer-loop related to the force equation, and the time-scale separation assumption is intermediately adopted here. In this step, it is assumed that the fast state has already achieved its desired value, without any considerations about its transient response (i.e. $z_{2}$ is zero).

Derivative of Lyapunov function candidate $V_{1}$ becomes

$$
\begin{aligned}
\dot{V}_{1} & =z_{1} \dot{z}_{1} \\
& =z_{1}\left(\hat{Z}_{\alpha}^{*} \alpha+q-\dot{\alpha}_{c}\right)
\end{aligned}
$$

In order to satisfy Lyapunov stability condition, a pseudocommand $q_{c}$ is derived as

$$
q_{c}=-C_{1} z_{1}-\hat{Z}_{\alpha}^{*} \alpha+\dot{\alpha}_{c}
$$

which makes negative definite $\dot{V}_{1}=-C_{1} z_{1}^{2}$ where $C_{1}$ is a positive design parameter. The state of the fast dynamics is regarded as a control input for the state of the slow dynamics.

Second, Lyapunov function candidate $V_{2}$ considering both $z_{1}$ and $z_{2}$ is selected as

$$
V_{2}=\frac{1}{2} z_{1}^{2}+\frac{1}{2} z_{2}^{2}
$$

which is positive definite. $V_{2}$ can be interpreted as an augmented $V_{1}$ with the additional term to penalize $z_{2}$, considering the transient response of the fast state. Another explanation about $V_{2}$ is also possible. For the inner-loop related to the moment equation, it's hard to assume that the slow state has already achieved its desired value (i.e. $z_{1}$ can't be assumed to be zero here). Hence, not only $z_{2}$, but also $z_{1}$ are considered in the inner-loop controller design. Regardless how this step is explained, the time-scale separation assumption is significantly relaxed, as a result.

Derivative of $V_{2}$ can be calculated as below.

$$
\begin{aligned}
\dot{V}_{2} & =z_{1} \dot{z}_{1}+z_{2} \dot{z}_{2} \\
& =z_{1}\left(\hat{Z}_{\alpha}^{*} \alpha+q-\dot{\alpha}_{c}\right)+z_{2}\left(\hat{M}_{\alpha}^{*} \alpha+\hat{M}_{q}^{*} q+\hat{M}_{\delta}^{*} \delta-\dot{q}_{c}\right)
\end{aligned}
$$

By using the pseudo-command (6), $\dot{V}_{2}$ becomes

$$
\dot{V}_{2}=z_{1}\left(-C_{1} z_{1}+z_{2}\right)+z_{2}\left(\hat{M}_{\alpha}^{*} \alpha+\hat{M}_{q}^{*} q+\hat{M}_{\delta}^{*} \delta-\dot{q}_{c}\right)
$$

To satisfy Lyapunov stability condition, the control command $\delta$ is derived as

$$
\delta=\frac{1}{\hat{M}_{\delta}^{*}}\left(-C_{2} z_{2}-z_{1}-\hat{M}_{\alpha}^{*} \alpha-\hat{M}_{q}^{*} q+\dot{q}_{c}\right)
$$

which makes negative definite $\dot{V}_{2}=-C_{1} z_{1}^{2}-C_{2} z_{2}^{2}$ where $C_{1}$ and $C_{2}$ are positive design parameters.

The final form of BKS controller derived in this subsection can be suggested as follows.

$$
\begin{aligned}
q_{c} & =-C_{1} z_{1}-\hat{Z}_{\alpha}^{*} \alpha+\dot{\alpha}_{c} \\
\delta & =\frac{1}{\hat{M}_{\delta}^{*}}\left(-C_{2} z_{2}-z_{1}-\hat{M}_{\alpha}^{*} \alpha-\hat{M}_{q}^{*} q+\dot{q}_{c}\right)
\end{aligned}
$$

The pseudo-command $q_{c}$ to make the angle of attack $\alpha$ achieve its desired value $\alpha_{c}$, is derived. $q$ goes to $q_{c}$ by the derived control input $\delta$. The model information about $\hat{Z}_{\alpha}^{*}, \hat{M}_{\delta}^{*}, \hat{M}_{\alpha}^{*}$ and $\hat{M}_{q}^{*}$ is required to implement the control algorithm.

\section{B. Incremental Backstepping Control}

For the outer-loop controller design, BKS, not IBKS, is utilized in this subsection. If IBKS is applied here, it additionally requires measurements about current state derivative and control in the outer-loop (i.e. $\dot{\alpha}_{0}$ and $q_{0}$ ). Instead of using those measurements hard to be obtained, more practical ways exist to compensate the model information $\hat{Z}_{\alpha}^{*}$. For example, more general measurements like a normal force and a velocity can directly substitute for $\hat{Z}_{\alpha}^{*} \alpha$. For this reason, the incremental law is not normally used for the outer-loop, and it can be seen in other papers [12] [14] [17] which just applied BKS or PID for it. In this paper, BKS is utilized for the outer-loop, to make the comparison easier with a pure BKS proposed in the previous subsection. Therefore, the first step is identically applied, so the pseudo-command becomes the same with (6).

For the inner-loop controller design, $q$ dynamics in (2) is modified assuming that the states $\alpha, q$ and the control input $\delta$ can be expressed as combinations of reference points $(\cdot)_{0}$ and 
disturbances $\Delta(\cdot)$ around them. This assumption is reasonable especially with a sufficiently high sampling rate.

$$
\begin{aligned}
\dot{q} & =\hat{M}_{\alpha}^{*}\left(\alpha_{0}+\Delta \alpha\right)+\hat{M}_{q}^{*}\left(q_{0}+\Delta q\right)+\hat{M}_{\delta}^{*}\left(\delta_{0}+\Delta \delta\right) \\
& =\dot{q}_{0}+\hat{M}_{\alpha}^{*} \Delta \alpha+\hat{M}_{q}^{*} \Delta q+\hat{M}_{\delta}^{*} \Delta \delta
\end{aligned}
$$

As in [12], [16], [19] and [20], the increments in states, $\Delta \alpha$ and $\Delta q$, can be ignored, since they have much smaller effects than the increment in input, $\Delta \delta$. This becomes acceptable as the control surface deflection directly and instantly affects the pitch moment, whereas the effect of $\Delta \alpha$ and $\Delta q$ on the pitch moment is not direct. Note that $\alpha$ and $q$ first create lift force and this force then induces the pitch moment. This implies that the effect of $\alpha$ and $q$ on the pitch moment is slower than that of $\delta$. Hence, comparing to the increment on control input, the effect of increments on state variables becomes negligible, especially when a sampling time is small enough. Then, $q$ dynamics for the inner-loop controller design with IBKS becomes

$$
\dot{q} \simeq \dot{q}_{0}+\hat{M}_{\delta}^{*} \Delta \delta
$$

In the second step, Lyapunov function candidate $V_{2}$ considering both $z_{1}$ and $z_{2}$ is selected as

$$
V_{2}=\frac{1}{2} z_{1}^{2}+\frac{1}{2} z_{2}^{2}
$$

which is positive definite. It can be interpreted in the same way with the previous subsection.

Derivative of $V_{2}$ can be calculated as below.

$$
\begin{aligned}
\dot{V}_{2} & =z_{1} \dot{z}_{1}+z_{2} \dot{z}_{2} \\
& =z_{1}\left(\hat{Z}_{\alpha}^{*} \alpha+q-\dot{\alpha}_{c}\right)+z_{2}\left(\dot{q}_{0}+\hat{M}_{\delta}^{*} \Delta \delta-\dot{q}_{c}\right)
\end{aligned}
$$

The only difference with (8) is that the dynamics (13) which is expressed into an incremental form is applied. By using the pseudo-command which is the same as $(6), \dot{V}_{2}$ becomes

$$
\dot{V}_{2}=z_{1}\left(-C_{1} z_{1}+z_{2}\right)+z_{2}\left(\dot{q}_{0}+\hat{M}_{\delta}^{*} \Delta \delta-\dot{q}_{c}\right)
$$

To satisfy Lyapunov stability condition, the control command $\Delta \delta$ is derived as

$$
\Delta \delta=\frac{1}{\hat{M}_{\delta}}\left(-C_{2} z_{2}-z_{1}-\dot{q}_{0}+\dot{q}_{c}\right)
$$

which makes negative definite $\dot{V}_{2}=-C_{1} z_{1}^{2}-C_{2} z_{2}^{2}$ where $C_{1}$ and $C_{2}$ are positive design parameters.

The final form of IBKS controller derived in this subsection can be suggested as follows.

$$
\begin{aligned}
q_{c} & =-C_{1} z_{1}-\hat{Z}_{\alpha}^{*} \alpha+\dot{\alpha}_{c} \\
\delta & =\delta_{0}+\Delta \delta \\
& =\frac{1}{\hat{M}_{\delta}^{*}}\left(-C_{2} z_{2}-z_{1}-\dot{q}_{0}+\dot{q}_{c}\right)+\delta_{0}
\end{aligned}
$$

Like BKS, $q$ goes to $q_{c}$ by $\delta$, and $\alpha$ goes to $\alpha_{c}$ by $q_{c}$. Comparing to pure BKS control command in the previous subsection, $\hat{M}_{\alpha}^{*}$ and $\hat{M}_{q}^{*}$ related terms are disappeared, because $\Delta \alpha$ and $\Delta q$ are neglected in $q$ dynamics (13) during the control law derivation. Only $\hat{Z}_{\alpha}^{*}$ and $\hat{M}_{\delta}^{*}$ are necessary for the implementation of the algorithm, so less model information is required. Instead of reduced model dependency, additional measurements $\delta_{0}$ and $\dot{q}_{0}$, current control surface deflection and state derivative, are required to implement the control algorithm.

\section{Closed-loop Analysis}

Closed-loop analysis is performed by substituting each control input (11) and (18) to the dynamics (1) with $Z_{\delta}^{*}=0$. In general, $C_{Z_{\delta}}$ is small enough to be neglected, especially for large aircrafts [26]. Comparing to the dynamics (2) utilized for the control law derivation, the main difference in the dynamics for the analysis is that the real aerodynamic derivatives, not the estimates, are considered. As in [22], analysis is performed in piece-wise way in order to easily utilize the existing analysis framework for linear time-invariant(LTI) system.

In this section, analysis starts with the nominal case, where transfer functions with both algorithms are derived assuming that the uncertainties do not exist in the aerodynamic derivative estimates. The next step is to consider the model uncertainties in the closed-loop analysis, so their effects to the systems with each controller can be investigated in stability and performance point of view. Under the model uncertainties, a condition to maintain stability and a steady state error are suggested for each closed-loop system. Although the control laws are designed to always ensure asymptotic stability with the positive design parameters in the nominal case, it cannot be guaranteed if real model information is different with the estimates utilized in the controller design phase due to the model uncertainties. Closed-loop characteristics with BKS and IBKS under the model uncertainties can be directly compared using derived common metric, which reinforces critical understandings about the algorithms. To have more insights from simplified situations, case studies are carried out, assuming that the uncertainty exists only in one aerodynamic derivative estimate and the other estimates have true values.

\section{A. Nominal Case}

Under the assumption of perfect model information without any uncertainties, ideal measurements without any delays, and constant $\alpha_{c}$ (i.e. $\dot{\alpha}_{c}=\ddot{\alpha}_{c}=0$ ), transfer functions with BKS and IBKS are derived for the nominal case. Their detailed derivation processes are addressed in Appendix A and B, and the results are suggested as (19).

$$
\begin{aligned}
& \frac{\alpha(s)}{\alpha_{c}(s)}=\frac{T_{N}}{s^{2}+2 \zeta_{N} \omega_{n_{N}} s+\omega_{n_{N}}^{2}} \\
& \text { where } \\
& T_{N}=C_{1} C_{2}+1 \\
& 2 \zeta_{N} \omega_{n_{N}}=C_{1}+C_{2} \\
& \omega_{n_{N}}^{2}=C_{1} C_{2}+1
\end{aligned}
$$

$\zeta_{N}$ and $\omega_{n_{N}}$ represent a damping ratio and a natural frequency for the closed-loop systems with BKS and IBKS in the nominal case. 
Poles with BKS and IBKS for the nominal case, $p_{N, 1}$ and $p_{N, 2}$, are given from (19) as follows.

$$
p_{N, 1}=p_{N, 2}=\frac{-\left(C_{1}+C_{2}\right) \pm \sqrt{\left(C_{1}-C_{2}\right)^{2}-4}}{2}
$$

From the equation (20) above, following properties can be identified. First, with the positive design parameters, poles are always located in left half plane, which means that the systems always become stable with BKS and IBKS. Second, poles can be expressed as a function of the design parameters only. This implies that the system characteristics become uniform in the entire flight envelope.

Steady state error, $e_{s s}$, can be calculated using following relationships.

$$
e_{s s}=\alpha_{c}-\lim _{t \rightarrow \infty} \alpha(t)=\alpha_{c}-\lim _{s \rightarrow 0} s \alpha(s)
$$

A step input $\alpha_{c}=K$ in time-domain is expressed in the frequency-domain as below.

$$
\alpha_{c}(s)=\frac{K}{s}
$$

By applying (19) and (22) to (21), steady state errors with BKS and IBKS for the nominal case, $e_{s s_{N}, 1}$ and $e_{s s_{N}, 2}$, can be calculated, resulting in zero values.

$$
e_{s s_{N}, 1}=e_{s s_{N}, 2}=0
$$

Because the transfer functions with both algorithms are suggested, various performance metrics can be discussed further. For instance, a settling time, when the magnitude of the state error is reduced within $5 \%$, can be calculated by the approximated equation (24) suggested below [27].

$$
t_{s_{N}}= \begin{cases}\frac{3.2}{\zeta_{N} \omega_{n_{N}}} & \text { if } 0<\zeta_{N}<0.69 \\ \frac{4.5}{\omega_{n_{N}}} \zeta_{N} & \text { if } \zeta_{N}>0.69\end{cases}
$$

where

$$
\begin{aligned}
\omega_{n_{N}} & =\sqrt{C_{1} C_{2}+1} \\
\zeta_{N} & =\frac{C_{1}+C_{2}}{2 \sqrt{C_{1} C_{2}+1}}
\end{aligned}
$$

This performance metric $t_{s_{N}}$, related to fast response, is also determined only by the design parameters $C_{1}$ and $C_{2}$.

For the nominal case, asymptotic stability is achieved in both closed-loop systems, which is expected in the design stage of the controllers. Although the incremental dynamics (13) is applied in the IBKS controller derivation process, the closed-loop analysis shows that the desired characteristics are accomplished, because the sampling rate is assumed to be fast enough for this sensor based approach.

\section{B. Closed-loop Analysis under the Model Uncertainties}

If the model uncertainties are considered, asymptotic stability cannot be achieved only with the positive design parameters, and performance cannot be uniform in whole flight envelope, unlike the nominal case. In this subsection, for each closed-loop system, a condition to maintain stability under the model uncertainties is investigated, along with performance metrics like a steady state error. To have more insights from simplified situations, case studies are carried out for each closed-loop system, assuming that the uncertainty exists only in one aerodynamic derivative estimate and the others have true values. Detailed description for each case is suggested in Table I, where $\Delta_{(\cdot)}$ denotes the uncertainty in the estimate for real aerodynamic derivative $(\cdot)$.

TABLE I

CASE DESCRIPTION

\begin{tabular}{|c||c|c|c|c|}
\hline & $\hat{M}_{\alpha}^{*}$ & $\hat{M}_{q}^{*}$ & $\hat{Z}_{\alpha}^{*}$ & $\hat{M}_{\delta}^{*}$ \\
\hline \hline Case 1 & $M_{\alpha}^{*}\left(1+\Delta_{M_{\alpha}^{*}}\right.$ & $M_{q}^{*}$ & $Z_{\alpha}^{*}$ & $M_{\delta}^{*}$ \\
\hline Case 2 & $M_{\alpha}^{*}$ & $M_{q}^{*}\left(1+\Delta_{M_{q}^{*}}\right)$ & $Z_{\alpha}^{*}$ & $M_{\delta}^{*}$ \\
\hline Case 3 & $M_{\alpha}^{*}$ & $M_{q}^{*}$ & $Z_{\alpha}^{*}\left(1+\Delta_{Z_{\alpha}^{*}}\right.$ & $M_{\delta}^{*}$ \\
\hline Case 4 & $M_{\alpha}^{*}$ & $M_{q}^{*}$ & $Z_{\alpha}^{*}$ & $M_{\delta}^{*}\left(1+\Delta_{M_{\delta}^{*}}\right.$ \\
\hline
\end{tabular}

1) Backstepping Control: If there exist the uncertainties in the aerodynamic derivative estimates, a transfer function with BKS can be derived as (26), under the assumption of perfect measurements without any delays and constant $\alpha_{c}$. Detailed derivation process is listed in Appendix A.

$$
\frac{\alpha(s)}{\alpha_{c}(s)}=\frac{T_{\Delta, 1}}{s^{2}+2 \zeta_{\Delta, 1} \omega_{n_{\Delta, 1}} s+\omega_{n_{\Delta, 1}}^{2}}
$$

where

$$
\begin{aligned}
& T_{\Delta, 1}= \frac{M_{\delta}^{*}}{\hat{M}_{\delta}^{*}}\left(C_{1} C_{2}+1\right) \\
& 2 \zeta_{\Delta, 1} \omega_{n_{\Delta, 1}}=-\left\{Z_{\alpha}^{*}+M_{q}^{*}-\frac{M_{\delta}^{*}}{\hat{M}_{\delta}^{*}}\left(C_{1}+C_{2}+\hat{M}_{q}^{*}+\hat{Z}_{\alpha}^{*}\right)\right\} \\
& \omega_{n_{\Delta, 1}}^{2}=\left\{\left(Z_{\alpha}^{*} M_{q}^{*}-M_{\alpha}^{*}\right)-\frac{M_{\delta}^{*}}{\hat{M}_{\delta}^{*}}\left(Z_{\alpha}^{*} \hat{M}_{q}^{*}-\hat{M}_{\alpha}^{*}\right)\right\} \\
&+\frac{M_{\delta}^{*}}{\hat{M}_{\delta}^{*}}\left\{C_{2}\left(\hat{Z}_{\alpha}^{*}-Z_{\alpha}^{*}\right)+\left(C_{1} C_{2}+1\right)\right\}
\end{aligned}
$$

$\zeta_{\Delta, 1}$ and $\omega_{n}$ represent a damping ratio and a natural frequency for the system with BKS under the model uncertainties. Because the model uncertainties are considered, not only $C_{1}$ and $C_{2}$, but also the aerodynamic derivatives and their estimates with the uncertainties, have impacts on the closedloop system and explicitly appear in the transfer function as (26), unlike (19) for the nominal case.

For a damped system, stability is normally guaranteed. Hence, a condition $\mathscr{G}_{\Delta, 1}$ to maintain stability for the system with BKS under the model uncertainties can be obtained from $2 \zeta_{\Delta, 1} \omega_{n_{\Delta, 1}}>0$ (Cond.1) under $\omega_{n_{\Delta, 1}}^{2}>0$ (Cond.2) as below. $\mathscr{G}_{\Delta, 1}=\left\{C_{1}, C_{2} \in \mathbb{R}_{>0} \mid\right.$ Cond. $1 \&$ Cond. 2 $\}$

Cond. $1: C_{1}+C_{2}>\left(\frac{\hat{M}_{\delta}^{*}}{M_{\delta}^{*}} Z_{\alpha}^{*}-\hat{Z}_{\alpha}^{*}\right)+\left(\frac{\hat{M}_{\delta}^{*}}{M_{\delta}^{*}} M_{q}^{*}-\hat{M}_{q}^{*}\right)$

Cond. 2: $\left(C_{1}+\hat{Z}_{\alpha}^{*}-Z_{\alpha}^{*}\right) C_{2}>-\frac{\hat{M}_{\delta}^{*}}{M_{\delta}^{*}}\left(Z_{\alpha}^{*} M_{q}^{*}-M_{\alpha}^{*}\right)$

$$
+\left(Z_{\alpha}^{*} \hat{M}_{q}^{*}-\hat{M}_{\alpha}^{*}\right)-1
$$


if $M_{\delta}^{*}$ and $\hat{M}_{\delta}^{*}$ have the same sign. This stability condition can be interpreted into constraints on the design parameters for BKS under the model uncertainties.

A steady state error $e_{s s_{A}, 1}$ with $\mathrm{BKS}$ controller can be obtained from (21), (22) and (26).

$$
e_{s s_{\Delta}, 1}=K \frac{\eta_{2}+\frac{\hat{M}_{\delta}^{*}}{M_{\delta}^{*}} \eta_{3}}{\eta_{1}+\eta_{2}+\frac{\hat{M}_{\delta}^{*}}{M_{\delta}^{*}} \eta_{3}}
$$

$\eta_{1}, \eta_{2}$ and $\eta_{3}$ in (28) can be written as follows.

$$
\begin{aligned}
& \eta_{1}=C_{1} C_{2}+1 \\
& \eta_{2}=C_{2}\left(\hat{Z}_{\alpha}^{*}-Z_{\alpha}^{*}\right) \\
& \eta_{3}=\left\{\left(Z_{\alpha}^{*} M_{q}^{*}-M_{\alpha}\right)-\frac{M_{\delta}^{*}}{\hat{M}_{\delta}^{*}}\left(Z_{\alpha}^{*} \hat{M}_{q}^{*}-\hat{M}_{\alpha}^{*}\right)\right\}
\end{aligned}
$$

$\eta_{1}$, the only difference between the numerator and the denominator in the derived steady state error equation (28), is dependent only upon the design parameters. $\eta_{2}$ is a function of the model information for the outer-loop and the design parameter for the inner-loop. $\eta_{3}$ is highly related to the model information for the inner-loop. Unlike the nominal case, $e_{s s_{\Delta}, 1}$ cannot be zero due to the model uncertainties. It can be reduced with high $C_{1}$, since $\eta_{1}$ increases as $C_{1}$ go up. It is difficult to generalize this property with the design parameter for the inner-loop $C_{2}$, because $\eta_{2}$ which is also affected by $C_{2}$, becomes effective to $e_{s s_{\Delta}, 1}$ when the uncertainty exists in $\hat{Z}_{\alpha}^{*}$. The steady state error becomes $\frac{\left(\hat{Z}_{\alpha}^{*}-Z_{\alpha}^{*}\right)}{C_{1}+\left(\hat{Z}_{\alpha}^{*}-Z_{\alpha}^{*}\right)}$ if $C_{2}$ goes to infinity, while $e_{s s_{A}, 1}$ goes to zero as $C_{1}$ goes to infinity.

To have better understandings about the effects of the uncertainty in each aerodynamic derivative estimate to the system with BKS, case studies are performed as below. Depending on where the uncertainty exists, even with the same level of the uncertainty, its impact to the closed-loop system can be different.

For each case, a transfer function with BKS (26) can be simplified into (30) with perturbed parameters $T^{\prime}{ }_{\Delta, 1}, 2 \zeta^{\prime}{ }_{\Delta, 1} \omega^{\prime}{ }_{n_{\Delta, 1}}$ and $\omega_{n_{\Delta, 1}}^{\prime 2}$ from the nominal $T_{N}, 2 \zeta_{N} \omega_{n_{N}}$, and $\omega_{n_{N}}^{2}$. These parameters are addressed in Table II.

$$
\frac{\alpha(s)}{\alpha_{c}(s)}=\frac{T_{\Delta, 1}^{\prime}}{s^{2}+2 \zeta^{\prime}{ }_{\Delta, 1} \omega_{n_{\Delta, 1}}^{\prime} s+\omega_{n_{\Delta, 1}}^{2}}
$$

TABLE II

CASE Study : Parameters FOr TRANSFER Function with BKS

\begin{tabular}{|c||c|c|c|}
\hline Case & $T^{\prime}{ }_{\Delta, 1}$ & $2 \zeta_{\Delta, 1}^{\prime} \omega^{\prime} n_{\Delta, 1}$ & $\omega_{n_{\Delta, 1}}^{\prime 2}$ \\
\hline \hline 1 & $T_{N}$ & $2 \zeta_{N} \omega_{n_{N}}$ & $\omega_{n_{N}}^{2}+M_{\alpha}^{*} \Delta_{M_{\alpha}^{*}}$ \\
\hline 2 & $T_{N}$ & $2 \zeta_{N} \omega_{n_{N}}+M_{q}^{*} \Delta_{M_{q}^{*}}$ & $\omega_{n_{N}}^{2}-Z_{\alpha}^{*} M_{q}^{*} \Delta_{M_{q}^{*}}$ \\
\hline 3 & $T_{N}$ & $2 \zeta_{N} \omega_{n_{N}}+Z_{\alpha}^{*} \Delta_{Z_{\alpha}^{*}}$ & $\omega_{n_{N}}^{2}+C_{2} Z_{\alpha}^{*} \Delta_{Z_{\alpha}^{*}}$ \\
\hline & & $\frac{2 \zeta_{N} \omega_{n_{N}}}{1+\Delta_{M_{\delta}^{*}}}$ & $\frac{\omega_{n_{N}}^{2}}{1+\Delta_{M_{\delta}^{*}}}$ \\
4 & $\frac{T_{N}}{1+\Delta_{M_{\delta}^{*}}}$ & $-\frac{\left(Z_{\alpha}^{*}+M_{q}^{*}\right) \Delta_{M_{\delta}^{*}}}{1+\Delta_{M_{\delta}^{*}}}$ & $+\frac{\left(Z_{\alpha}^{*} M_{q}^{*}-M_{\alpha}^{*}\right) \Delta_{M_{\delta}^{*}}}{1+\Delta_{M_{\delta}^{*}}}$ \\
& & & \\
\hline
\end{tabular}

Note that, except the case $4, T^{\prime}{ }_{\Delta, 1}$ becomes the same as the nominal one, and the additive perturbation term appears in the parameters $2 \zeta^{\prime}{ }_{\Delta, 1} \omega^{\prime} n_{\Delta, 1}$ and $\omega^{\prime 2}{ }_{n}$ respectively. This perturbation term can be described as a product of the weight factor and the uncertainty $\Delta_{(\cdot)}$. The parameters for the case 4 show similar forms with the others, but they are additionally divided by $\left(1+\Delta_{M_{\delta}^{*}}\right)$.

For each case, using the parameters $2 \zeta^{\prime}{ }_{\Delta, 1} \omega^{\prime}{ }_{n_{\Delta, 1}}$ and $\omega^{\prime 2} n_{\Delta, 1}$ in Table I, a stability condition can be simplified into (31) with Table III.

$$
\begin{aligned}
& \mathscr{G}_{\Delta, 1}^{\prime}=\left\{C_{1}, C_{2} \in \mathbb{R}_{>0} \mid \text { Cond. } 1 \& \text { Cond. } 2\right\} \\
& \text { Cond. } 1: C_{1}+C_{2}>\kappa_{1,1}
\end{aligned}
$$

Cond. 2 : $C_{1} C_{2}+1>\kappa_{2,1}$

TABLE III

CASE Study : Parameters For Stability Conditions with BKS

\begin{tabular}{|c||c|c|}
\hline Case & $\kappa_{1,1}$ & $\kappa_{2,1}$ \\
\hline \hline 1 & 0 & $-M_{\alpha}^{*} \Delta_{M_{\alpha}^{*}}$ \\
\hline 2 & $-M_{q}^{*} \Delta_{M_{q}^{*}}$ & $Z_{\alpha}^{*} M_{q}^{*} \Delta_{M_{q}^{*}}$ \\
\hline 3 & $-Z_{\alpha}^{*} \Delta_{Z_{\alpha}^{*}}$ & $-C_{2} Z_{\alpha}^{*} \Delta_{Z_{\alpha}^{*}}$ \\
\hline 4 & $\left(Z_{\alpha}^{*}+M_{q}^{*}\right) \Delta_{M_{\delta}^{*}}$ & $-\left(Z_{\alpha}^{*} M_{q}^{*}-M_{\alpha}^{*}\right) \Delta_{M_{\delta}^{*}}$ \\
\hline
\end{tabular}

$\kappa_{1,1}$ and $\kappa_{2,1}$, which are related to the feasible boundary of the design parameters, appear to be the perturbation term of $2 \zeta^{\prime}{ }_{\Delta, 1} \omega_{n_{\Delta, 1}}^{\prime}$ and $\omega_{n_{\Delta, 1}^{\prime}}^{2}$ with the opposite sign. Depending on where the uncertainty exists, the boundary value of each $\Delta_{(\cdot)}$ for the stable closed-loop system can be different. It becomes small if the corresponding weight factor is large. It is observed from Table III that, for the case 1, Cond.1 is always satisfied with the positive design parameters, so only Cond.2 is effective.

By comparing the cases, more simplified structure for the steady state error equation can be found as (32). $\eta_{1}$ in (32) is identical with the one in (28), defined only by the design parameters. For each case, the weight factor to the model uncertainty $\eta_{2,1}^{\prime}$ only changes, and it is listed in Table IV.

$$
e_{s s_{\Delta}, 1}^{\prime}=K \frac{\eta_{2,1}^{\prime} \Delta_{(\cdot)}}{\eta_{1}+\eta_{2,1}^{\prime} \Delta_{(\cdot)}}
$$

TABLE IV

Case Study : Parameters for Steady State ERror with B KS

\begin{tabular}{|c||c|}
\hline Case & $\eta^{\prime}{ }_{2,1}$ \\
\hline \hline 1 & $M_{\alpha}^{*}$ \\
\hline 2 & $-Z_{\alpha}^{*} M_{q}^{*}$ \\
\hline 3 & $C_{2} Z_{\alpha}^{*}$ \\
\hline 4 & $Z_{\alpha}^{*} M_{q}^{*}-M_{\alpha}^{*}$ \\
\hline
\end{tabular}

As can be seen in Table III and IV, $\kappa_{2,1}=-\eta^{\prime}{ }_{2,1} \Delta_{(\cdot)}$. Depending on where the uncertainty exists, even with the same level of the uncertainty, $\left|e_{s_{s_{\Delta}}, 1}\right|$ gets larger for the case with greater weight factor. Additionally, $\left|e_{s s_{\Delta}, 1}\right|$ goes up as $\left|\Delta_{(\cdot)}\right|$ increases.

2) Incremental Backstepping Control: For successful analysis with IBKS, it is necessary to decide how to deal with the additional measurements $\dot{q}_{0}$ and $\delta_{0}$. In this paper, they are suggested as follows.

$$
\begin{aligned}
& \dot{q}_{0}=M_{\alpha}^{*} \alpha+M_{q}^{*} q+M_{\delta}^{*} \delta_{0} \\
& \delta_{0}=\delta(t-\tau)
\end{aligned}
$$


The model for the current state derivative measurement $\dot{q}_{0}$ comes from the piece-wise version of (1). For an ideal actuator, a control surface deflection directly follows a generated control command. Then, the current control surface deflection measurement $\delta_{0}$ can be regarded as a control command generated in the previous step, where $\tau$ indicates a step size.

If the uncertainties exist in the aerodynamic derivative estimates, a transfer function with IBKS can be obtained as (34), under the assumption of perfect measurements without any delays and constant $\alpha_{c}$. Detailed derivation process is addressed in Appendix B.

$$
\frac{\alpha(s)}{\alpha_{c}(s)}=\frac{D_{\Delta, 2}(s)}{s^{2}+N_{\Delta, 2}(s) s+N_{\Delta, 2}^{\prime}(s)}
$$

where

$$
\begin{aligned}
D_{\Delta, 2}(s)= & \frac{M_{\delta}^{*}}{\phi(s)}\left(C_{1} C_{2}+1\right) \\
N_{\Delta, 2}(s)= & -\left\{Z_{\alpha}^{*}+M_{q}^{*}-\frac{M_{\delta}^{*}}{\phi(s)}\left(C_{1}+C_{2}+M_{q}^{*}+\hat{Z}_{\alpha}^{*}\right)\right\} \\
N_{\Delta, 2}^{\prime}(s)= & \left(1-\frac{M_{\delta}^{*}}{\phi(s)}\right)\left(Z_{\alpha}^{*} M_{q}^{*}-M_{\alpha}^{*}\right) \\
& +\frac{M_{\delta}^{*}}{\phi(s)}\left\{C_{2}\left(\hat{Z}_{\alpha}^{*}-Z_{\alpha}^{*}\right)+\left(C_{1} C_{2}+1\right)\right\}
\end{aligned}
$$

$\phi(s)$ is defined as below.

$$
\phi(s)=\hat{M}_{\delta}^{*}\left(1-e^{-\tau s}\right)+M_{\delta}^{*} e^{-\tau s}
$$

Since the model uncertainties are considered, not only $C_{1}$ and $C_{2}$, but also the aerodynamic derivatives and their estimates with the uncertainties, have impacts on the closed-loop system and explicitly appear in the transfer function as (34), unlike (19) for the nominal case. Comparing to (26) with BKS, there are the two main differences in the transfer function (34). First, the true values for the stability derivatives in the inner-loop, not the estimated ones, appear in (34). Second, the effect of the control derivative estimate $\hat{M}_{\delta}^{*}$ resides in $\phi(s)$.

The transfer function with IBKS can be simplified as (36) with $\tau \simeq 0$ assumption for the analysis purpose. This is reasonable assumption thanks to improved computation power and reduced transmission time in recent avionics systems.

$$
\begin{aligned}
& \frac{\alpha(s)}{\alpha_{c}(s)}=\frac{T_{\Delta, 2}}{s^{2}+2 \zeta_{\Delta, 2} \omega_{n_{\Delta, 2}} s+\omega_{n_{\Delta, 2}}^{2}} \\
& \text { where } \\
& T_{\Delta, 2}=C_{1} C_{2}+1 \\
& 2 \zeta_{\Delta, 2} \omega_{n_{\Delta, 2}}=\left(C_{1}+C_{2}\right)+\left(\hat{Z}_{\alpha}^{*}-Z_{\alpha}^{*}\right) \\
& \omega_{n_{\Delta, 2}}^{2}=\left(C_{1} C_{2}+1\right)+C_{2}\left(\hat{Z}_{\alpha}^{*}-Z_{\alpha}^{*}\right)
\end{aligned}
$$

$\zeta_{\Delta, 2}$ and $\omega_{n}$ denote a damping ratio and a natural frequency for the system with IBKS under the model uncertainties. An interesting observation from (36) is that, under the $\tau \simeq 0$ assumption, the effects of $M_{\delta}^{*}$ and its estimate with the uncertainty are vanished in the closed-loop system with IBKS, while they still remain in the system with BKS. This implies that, if the control command is calculated, transmitted and reflected fast enough to the real control surface deflection, the closed-loop system with IBKS becomes insensitive to the uncertainty in $\hat{M}_{\delta}^{*}$, although this information is still required to implement the control algorithm.

The differences in the analysis results with IBKS to the ones with BKS, can be explained as follows. The incremental controller for the inner-loop utilizes the additional measurements $\dot{q}_{0}$ and $\delta_{0}$. In $\dot{q}_{0}$ measurement obtained in flight, the effects of true $M_{\alpha}^{*}$ and $M_{q}^{*}$ are included in implicit way. Hence, model information about $M_{\alpha}^{*}$ and $M_{q}^{*}$ is not required for IBKS, unlike BKS. Not only these stability derivatives, but also the control derivative $M_{\delta}^{*}$ are involved in this $\dot{q}_{0}$ measurement. If there is no uncertainty in $\hat{M}_{\delta}^{*}$ utilized to implement the algorithm, the effect of $M_{\delta}^{*}$ in the $\dot{q}_{0}$ measurement can be totally compensated with the $\delta_{0}$ measurement, in the suggested control law (18). Generally, they cannot be fully cancelled out due to the uncertainty, resulting in $\phi(s)$ for the closedloop system with IBKS. However, if $\tau \simeq 0, \phi(s)$ becomes just $M_{\delta}^{*}$, even if $\hat{M}_{\delta}^{*}$ has uncertainty. Hence, the closedloop system with IBKS becomes robust with respect to the uncertainty in $\hat{M}_{\delta}^{*}$ utilized to implement the algorithm, if the control command is calculated, transmitted and reflected fast enough to the real control surface deflection. To sum up, all model uncertainties do not affect the system with IBKS when computation and transmission of the control command are fast enough. This is the key difference in the system behaviors with IBKS, comparing to the ones with BKS.

For the same reason in the subsection above with BKS, a condition $\mathscr{G}_{\Delta, 2}$ to maintain stability for the system with IBKS under the model uncertainties can be obtained from $2 \zeta_{\Delta, 2} \omega_{n_{\Delta, 2}}>0$ (Cond.1) under $\omega_{n_{\Delta, 2}}^{2}>0$ (Cond.2) as below.

$$
\begin{aligned}
& \mathscr{G}_{\Delta, 2}=\left\{C_{1}, C_{2} \in \mathbb{R}_{>0} \mid \text { Cond. } 1 \& \text { Cond. } 2\right\} \\
& \text { Cond. } 1: C_{1}+C_{2}>-\left(\hat{Z}_{\alpha}^{*}-Z_{\alpha}^{*}\right) \\
& \text { Cond. } 2:\left(C_{1}+\hat{Z}_{\alpha}^{*}-Z_{\alpha}^{*}\right) C_{2}>-1
\end{aligned}
$$

This stability condition can be interpreted into constraints on the design parameters for IBKS under the model uncertainties. Note that the stability condition is affected only by the uncertanty on $\hat{Z}_{\alpha}^{*}$ utilized for the outer-loop controller design with BKS.

Through the same process above using (21), (22) and (36), a steady state error $e_{s s_{\Delta}, 2}$ with IBKS controller can be suggested as follows.

$$
e_{s_{\Delta}, 2}=K \frac{\eta_{2}}{\eta_{1}+\eta_{2}}
$$

where

$$
\begin{aligned}
& \eta_{1}=C_{1} C_{2}+1 \\
& \eta_{2}=C_{2}\left(\hat{Z}_{\alpha}^{*}-Z_{\alpha}^{*}\right)
\end{aligned}
$$

Unlike the nominal case, the steady state error exist as (38) with (39) due to the model uncertainties. Comparing to (28) with (29) for pure BKS, there exist the same $\eta_{1}$ and $\eta_{2}$ in (38) with (39) for IBKS. However, there is no $\eta_{3}$ which is mainly related to the model information for the inner-loop, unlike pure BKS. The steady state error occurs only when there 
exists uncertainty on $\hat{Z}_{\alpha}^{*}$ utilized for the outer-loop controller design with BKS. In both (28) and (38), the denominator is bigger than the numerator by $\eta_{1}$ which increases as the design parameters go up. It indicates that high $C_{1}$ can also reduce $e_{s s, 2}$. For the same reason in the subsection above with pure $\mathrm{BKS}$, it is difficult to generalize this property with the design parameter for the inner-loop $C_{2}$.

Case studies are conducted to clearly show the effects of the uncertainty in each aerodynamic derivative estimate to the system with IBKS. Since $\hat{M}_{\alpha}^{*}$ and $\hat{M}_{q}^{*}$ are not required to implement IBKS thanks to the additional $\dot{q}_{0}$ measurement, the closed-loop system with IBKS is not affected by $\Delta_{M_{\alpha}^{*}}$ and $\Delta_{M_{q}^{*}}$. Hence, the analysis results with IBKS for the case 1 and 2 are the same with the ones for the nominal case. In addition, under the $\tau \simeq 0$ assumption, the analysis results with IBKS for the case 4 also become identical to the ones for the nominal case. It is shown that the closed-loop system with IBKS becomes robust with respect to $\Delta_{M_{\delta}^{*}}$, if the control command is calculated, transmitted and reflected fast enough to the real control surface deflection. For the case 3 where the model uncertainty only exists in dynamics for the outer-loop, the analysis results with IBKS appear to be the same with the corresponding case study results with pure BKS in the previous subsection. This can be explained from the fact that each outer-loop controller is designed with BKS in common, and inner-loop controllers with BKS and IBKS become the same when there are no model uncertainties in dynamics for the inner-loop, which is the case 3 .

\section{Simulation}

In this chapter, simulations are performed to verify the proposed theoretical analysis results. Since a piece-wise approach is considered to handle the LPV system as in [22], several points assigned to each grid were simulated. The aerodynamic derivatives shown in Fig. 7 of Appendix $\mathrm{C}$ and zero $Z_{\delta}^{*}$ are utilized for the simulations. As an example, simulation results when altitude is $7.6200 \mathrm{~km}$ and $U_{0}=185.9280 \mathrm{~m} / \mathrm{s}$ are suggested in this paper.

Simulation parameters about the angle of attack command, the design parameters, and the level of the model uncertainties are suggested in Table $\mathrm{V}$. The simulation parameter $\Delta_{(\cdot)}$ which represents the level of the model uncertainty, indicates how much percentage of error exists in the aerodynamic derivative estimate utilized in the control system design, comparing to the actual aerodynamic derivative $(\cdot)$. For example, $\Delta_{(\cdot)}=-0.75$ means that an aerodynamic derivative estimate used in the flight controller is $75 \%$ smaller than its real value for $(\cdot)$. $\Delta_{(\cdot)}=1$ implies that the flight controller utilizes an aerodynamic derivative estimate twice larger than its real value for $(\cdot)$. Initial values for the state variables $\alpha$ and $q$ are set to be $0^{\circ}$ and $0^{\circ} / \mathrm{s}$. Small enough $\tau$ is applied as $0.001 \mathrm{sec}$ for the simulation. With BKS, simulations are carried out for case 1 , 2, 3 and 4 respectively. With IBKS, simulations are performed for each case 3 and 4 .

The stability condition and the performance metric like $e_{s s}$ predicted by the analysis, can be examined in pole trajectories and time response graphs. Besides, simulation results show
TABLE V

SIMULATION PARAMETERS

\begin{tabular}{|c|c|}
\hline Parameter & Value \\
\hline \hline$\alpha_{c}$ & $1.5^{\circ}$ \\
\hline$C_{1}, C_{2}$ & 1.5 \\
\hline$\Delta_{(\cdot)}$ & {$[-0.75,-0.5,-0.25,0,0.25,0.5,0.75,1]$} \\
\hline
\end{tabular}

which model uncertainty has much influence in stability and performance for this aircraft.

\section{A. Simulation results with $B K S$}

Fig. 1 shows that the system becomes unstable when $\Delta_{M_{\alpha}^{*}}=0.75$ and 1. From Fig. 2, it is observed that $\Delta_{M_{q}^{*}}=$ $0.5,0.75$ and 1 can result in unstable behaviors of the system. Fig. 3 indicates that the closed-loop system is stable with every $\Delta_{Z_{\alpha}^{*}}$. It can be seen from Fig. 4 that $\Delta_{M_{\delta}^{*}}=-0.5$ and -0.75 can make the system unstable.

These observations can be clearly explained by the stability condition (31) with Table III. Under this simulation environment, the Cond. 2 in (31) is appeared to be a dominant condition for stability. The Cond. 2 implies that, for larger $\left|\eta^{\prime}{ }_{2,1}\right|$ case, the closed-loop system becomes unstable with smaller $\left|\Delta_{(\cdot)}\right| \cdot \eta_{2,1}^{\prime}=-4.7488,-7.7182,-2.9439$ and 12.4670 for Case 1, 2, 3 and 4 , respectively. Thus, $\left|\Delta_{(\cdot)}\right|$ resulting in unstable behavior to the system is predicted to be smaller for the Case 4 than for the Case 2, 1 and 3 where it becomes the largest. This coincides with the observations above obtained from Fig. 1 to 4 .

By rewriting the stability condition (31), a minimum $C_{1}$ to guarantee stability for each case under fixed $C_{2}$ and every $\left|\Delta_{(\cdot)}\right|$ in this simulation, can be predicted. It is expected that, in the stability point of view, $C_{1}>2.4992$ for the Case 1, $C_{1}>4.4788$ for the Case $2, C_{1}>1.2960$ for the Case 3 , and $C_{1}>5.5668$ for the Case 4 , under this simulation environment. Thus, all simulations with the BKS in this paper for every case will show stable results, by increasing $C_{1}$ larger than 5.5668, which is the minimum $C_{1}$ for the Case 4 where $\left|\eta_{2,1}^{\prime}\right|$ is the largest.

Steady state errors $e_{s s, 1}$ are given in Table VI for stable instances. It is interpreted from (32) that, $\left|e_{s s, 1}\right|$ is larger for the case with greater $\left|\eta_{2,1}^{\prime}\right|$, under the same $\left|\Delta_{(\cdot)}\right|$. The comparison should be carefully carried out between the cases with the same sign of $\eta_{2,1}^{\prime} \Delta_{(\cdot)} . \eta_{2,1}^{\prime}=-4.7488,-7.7182$, -2.9439 and 12.4670 for the Case 1, 2, 3 and 4, respectively. Thus, $\left|\eta_{2,1}^{\prime}\right|$ is predicted to be smaller for the Case 3 than for the Case 1, 2 and 4 where it becomes the largest. The sign of $\eta_{2,1}^{\prime}$ is positive only for the Case 4 and it is negative for the other three cases. Thus, the Case 1, 2 and 3 with $\Delta_{(\cdot)}$ and the Case 4 with $-\Delta_{(\cdot)}$ should be compared with each other. By applying this comparison scheme to Table VI, the interpretation from (32) is shown to agree with the simulation results.

Additionally, it is observed from Table VI that $\left|e_{s s, 1}\right|$ becomes larger as $\left|\Delta_{(\cdot)}\right|$ grows, which coincides with the analysis result. 


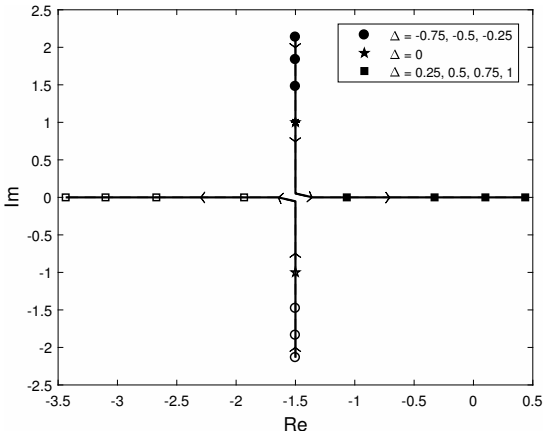

(a) Pole Trajectory

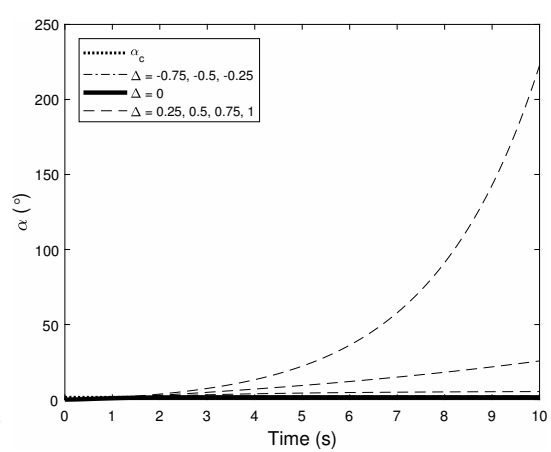

(b) Time Response

Fig. 1. Closed-loop System Response with BKS under the uncertainty in $\hat{M}_{\alpha}^{*}$

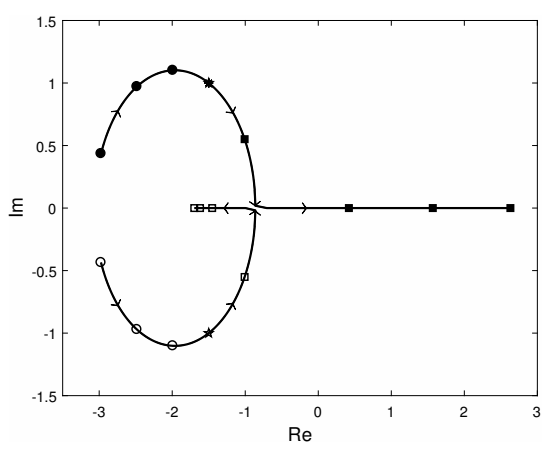

(a) Pole Trajectory

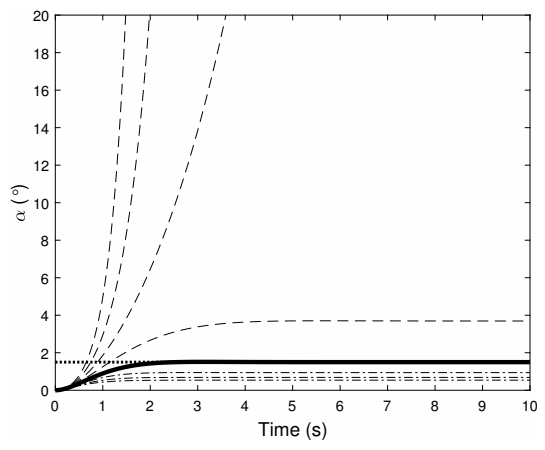

(b) Time Response

Fig. 2. Closed-loop System Response with BKS under the uncertainty in $\hat{M}_{q}^{*}$

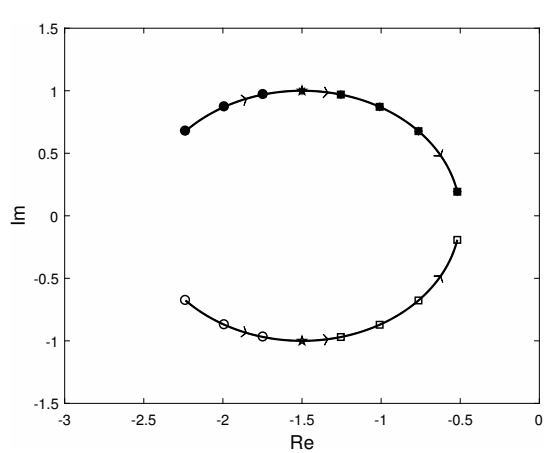

(a) Pole Trajectory

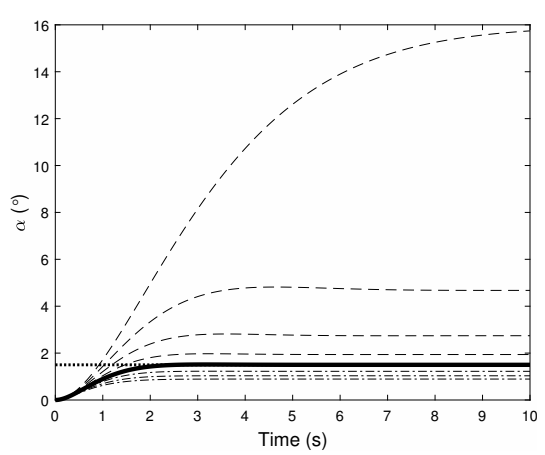

(b) Time Response

Fig. 3. Closed-loop System Response with BKS under the uncertainty in $\hat{Z}_{\alpha}^{*}$

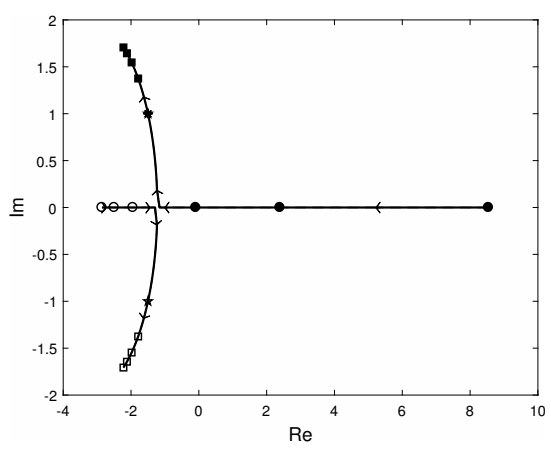

(a) Pole Trajectory

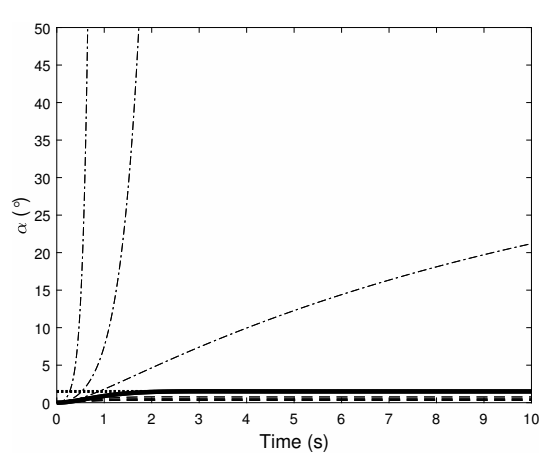

(b) Time Response

Fig. 4. Closed-loop System Response with BKS under the uncertainty in $\hat{M}_{\delta}^{*}$ 
TABLE VI

STEAdy STATE ERROR FOR SYSTEM WITH BKS, $e_{s, 1}$

\begin{tabular}{|c||c|c|c|c|c|c|c|c|}
\hline Case & -0.75 & -0.5 & -0.25 & 0 & 0.25 & 0.5 & 0.75 & 1 \\
\hline \hline 1 & 0.7843 & 0.6332 & 0.4013 & 0 & -0.8632 & -3.8281 & - & - \\
\hline 2 & 0.9606 & 0.8143 & 0.5588 & 0 & -2.1919 & - & - & - \\
\hline 3 & 0.6068 & 0.4676 & 0.2770 & 0 & -0.4391 & -1.2418 & -3.1749 & -14.4279 \\
\hline 4 & - & - & -35.0820 & 0 & 0.7343 & 0.9859 & 1.1131 & 1.1898 \\
\hline
\end{tabular}

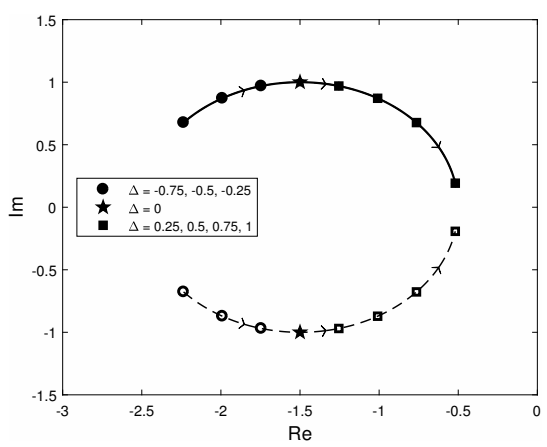

(a) Pole Trajectory

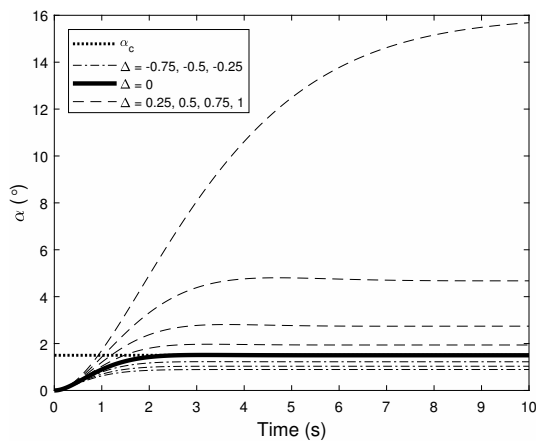

(b) Time Response

Fig. 5. Closed-loop System Response with IBKS under the uncertainty in $\hat{Z}_{\alpha}^{*}$

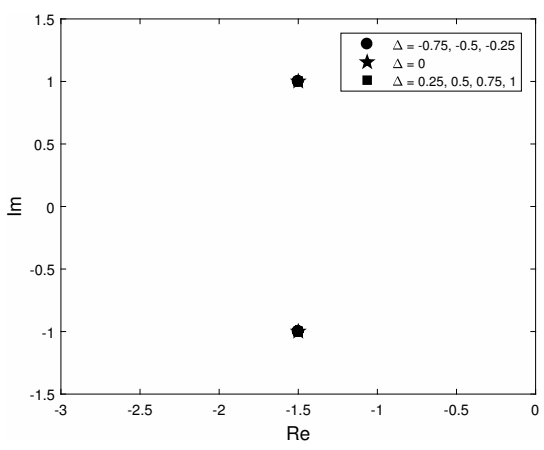

(a) Pole Trajectory

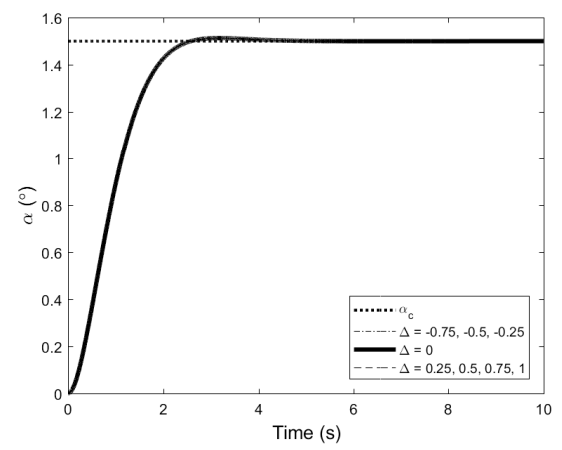

(b) Time Response

Fig. 6. Closed-loop System Response with IBKS under the uncertainty in $\hat{M}_{\delta}^{*}$

\section{B. Simulation results with IBKS}

Fig. 5 shows the identical results with Fig. 3. For case 3 , the system behaviors with IBKS and BKS become the same, which has been already predicted and explained in the analysis part. Under this simulation environment, the closedloop system is stable with every $\Delta_{z_{\alpha}^{*}} \cdot\left|e_{s s, 1}\right|$ increases as $\left|\Delta_{z_{\alpha}^{*}}\right|$ gets larger, and the system response becomes slower as $\Delta_{Z_{\alpha}^{*}}$ rises.

Fig. 6 shows that the closed-loop system is insensitive to the model uncertainty $\Delta_{M_{\delta}^{*}}$ in $\hat{M}_{\delta}^{*}$, with $\tau=0.001$ which is set to be small enough for the simulation. Poles are always in the left half plane, so the system is stable all the time. There is no change in the location of poles depending on the variation of $\Delta_{M_{\delta}^{*}}$. Consequently, $\omega_{n}, \zeta$ and $t_{s}$ remain the same, and $e_{s s, 2}$ doesn't exist. These simulation results coincide with the prediction from the analysis under the assumption that the control command is calculated, transmitted and reflected fast enough to the real control surface deflection.

\section{CONCLUSION}

This paper suggests closed-loop analysis results with BKS and IBKS methods under model uncertainties. The proposed analysis enables critical understandings and insights about system characteristics under the model uncertainties. Transfer functions with BKS and IBKS under the model uncertainties are compared with the ones for the nominal case. The effects of the model uncertainties on the closed-loop systems are figured out, resulting in the condition to maintain stability and the steady state error. The closed-loop characteristics with BKS and IBKS under the model uncertainties are directly compared using derived common metrics, which clarifies how the effects of the model uncertainties to the closed-loop system become different depending on the applied control algorithm. Unlike BKS, IBKS is not affected by the uncertainties on any model parameters, including control effectiveness which is still required for IBKS implementation, when the control input is calculated, transmitted and reflected fast enough to the actual 
control surface. Under the assumption that the uncertainty exists only in one aerodynamic derivative estimate while the other estimates have true values, case studies are conducted to find the impact of the uncertainty on the specific aerodynamic derivative estimate to the closed-loop system. Depending on where the uncertainty exists, even with the same level of the uncertainty, its impact to the closed-loop system becomes different, and this is explained with the weight factors for each case. As a future work, the short period mode dynamics can be extended to full 6-DoF dynamics.

\section{APPENDIX A}

\section{DERIVATION OF TRANSFER FUNCTION WITH BKS}

Dynamics (1) with $Z_{\delta}^{*}=0$ can be expressed as a state space equation below.

$$
\begin{aligned}
& \dot{\mathbf{x}}=\mathbf{A x}+\mathbf{B u} \quad \mathbf{y}=\mathbf{C x} \\
& \mathbf{x}=\left[\begin{array}{ll}
\alpha & q
\end{array}\right]^{T} \quad \mathbf{u}=\delta \\
& \mathbf{A}=\left[\begin{array}{cc}
Z_{\alpha}^{*} & 1 \\
M_{\alpha}^{*} & M_{q}^{*}
\end{array}\right] \quad \mathbf{B}=\left[\begin{array}{c}
0 \\
M_{\delta}^{*}
\end{array}\right] \quad \mathbf{C}=\left[\begin{array}{ll}
1 & 0
\end{array}\right]
\end{aligned}
$$

Using (11) with (1) and (3) under the assumption of constant $\alpha_{c}$ and zero $Z_{\delta}^{*}, \delta$ can be rearranged as follows.

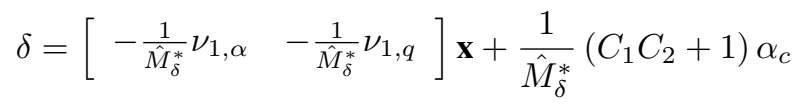

where

$\nu_{1, \alpha}=\left\{\left(C_{1}+\hat{Z}_{\alpha}^{*}\right)\left(C_{2}+Z_{\alpha}^{*}\right)+\hat{M}_{\alpha}^{*}+1\right\}$

$\nu_{1, q}=\left(C_{1}+C_{2}+\hat{M}_{q}^{*}+\hat{Z}_{\alpha}^{*}\right)$

Closed-loop system with BKS can be derived by substituting (41) into (40).

$$
\begin{aligned}
\dot{\mathbf{x}} & =\mathbf{A}_{1} \mathbf{x}+\mathbf{B}_{1} \alpha_{c} \quad \mathbf{y}=\mathbf{C} \mathbf{x} \\
\mathbf{A}_{1} & =\left[\begin{array}{ll}
a_{1,11} & a_{1,12} \\
a_{1,21} & a_{1,22}
\end{array}\right] \\
& =\left[\begin{array}{cc}
Z_{\alpha}^{*} & 1 \\
M_{\alpha}^{*}-\frac{M_{\delta}^{*}}{\hat{M}_{\delta}^{*}} \nu_{1, \alpha} & M_{q}^{*}-\frac{M_{\delta}^{*}}{\hat{M}_{\delta}^{*}} \nu_{1, q}
\end{array}\right] \\
\mathbf{B}_{1} & =\left[\begin{array}{c}
0 \\
\frac{M_{\delta}^{*}}{\hat{M}_{\delta}^{*}}\left(C_{1} C_{2}+1\right)
\end{array}\right] \\
\mathbf{C} & =\left[\begin{array}{ll}
1 & 0
\end{array}\right]
\end{aligned}
$$

Transfer function for the closed-loop system with BKS can be derived as below.

$$
\begin{aligned}
\frac{\alpha(s)}{\alpha_{c}(s)} & =\mathbf{C}(s)\left(s \mathbf{I}-\mathbf{A}_{1}(s)\right)^{-1} \mathbf{B}_{1}(s) \\
& =\frac{a_{1,12} \frac{M_{\delta}^{*}}{\hat{M}_{\delta}^{*}}\left(C_{1} C_{2}+1\right)}{s^{2}-\left(a_{1,11}+a_{1,22}\right) s+\left(a_{1,11} a_{1,22}-a_{1,12} a_{1,21}\right)} \\
& =\frac{T(s)}{s^{2}+2 \zeta \omega_{n} s+\omega_{n}^{2}}
\end{aligned}
$$

where

$$
\begin{aligned}
T(s)= & a_{1,12} \frac{M_{\delta}^{*}}{\hat{M}_{\delta}^{*}}\left(C_{1} C_{2}+1\right)=\frac{M_{\delta}^{*}}{\hat{M}_{\delta}^{*}}\left(C_{1} C_{2}+1\right) \\
2 \zeta \omega_{n}= & -\left(a_{1,11}+a_{1,22}\right) \\
= & -\left\{Z_{\alpha}^{*}+M_{q}^{*}-\frac{M_{\delta}^{*}}{\hat{M}_{\delta}^{*}}\left(C_{1}+C_{2}+\hat{M}_{q}^{*}+\hat{Z}_{\alpha}^{*}\right)\right\} \\
\omega_{n}^{2}= & a_{1,11} a_{1,22}-a_{1,12} a_{1,21} \\
= & \left\{\left(Z_{\alpha}^{*} M_{q}^{*}-M_{\alpha}^{*}\right)-\frac{M_{\delta}^{*}}{\hat{M}_{\delta}^{*}}\left(Z_{\alpha}^{*} \hat{M}_{q}^{*}-\hat{M}_{\alpha}^{*}\right)\right\} \\
& +\frac{M_{\delta}^{*}}{\hat{M}_{\delta}^{*}}\left\{C_{2}\left(\hat{Z}_{\alpha}^{*}-Z_{\alpha}^{*}\right)+\left(C_{1} C_{2}+1\right)\right\}
\end{aligned}
$$

When the uncertainties in every aerodynamic derivative estimate are neglected in (43), the closed-loop transfer function with BKS for the nominal case can be obtained as (19).

\section{APPENDIX B \\ DERIVATION OF TRANSFER FUNCTION WITH IBKS}

Using (18) with (1), (3) and (33) under the assumption of constant $\alpha_{c}$ and zero $Z_{\delta}^{*}, \delta$ can be rearranged as follows.

$$
\begin{aligned}
\delta= & -\frac{1}{\hat{M}_{\delta}^{*}} \nu_{2, \alpha} \alpha-\frac{1}{\hat{M}_{\delta}^{*}} \nu_{2, q} q+\frac{1}{\hat{M}_{\delta}^{*}}\left(C_{1} C_{2}+1\right) \alpha_{c} \\
& +\left(1-\frac{M_{\delta}^{*}}{\hat{M}_{\delta}^{*}}\right) \delta(t-\tau)
\end{aligned}
$$

where

$$
\begin{aligned}
& \nu_{2, \alpha}=\left\{\left(C_{1}+\hat{Z}_{\alpha}^{*}\right)\left(C_{2}+Z_{\alpha}^{*}\right)+M_{\alpha}^{*}+1\right\} \\
& \nu_{2, q}=\left(C_{1}+C_{2}+M_{q}^{*}+\hat{Z}_{\alpha}^{*}\right)
\end{aligned}
$$

Applying Laplace transform to (44) and arranging the equation with respect to $\delta$,

$$
\begin{aligned}
\delta(s)= & {\left[-\frac{1}{\phi(s)} \nu_{2, \alpha}(s)-\frac{1}{\phi(s)} \nu_{2, q}(s)\right] \mathbf{X}(s) } \\
& +\frac{1}{\phi(s)}\left(C_{1} C_{2}+1\right) \alpha_{c}(s)
\end{aligned}
$$

where

$$
\phi(s)=\hat{M}_{\delta}^{*}\left(1-e^{-\tau s}\right)+M_{\delta}^{*} e^{-\tau s}
$$

Dynamics for closed-loop analysis with IBKS is the same with (40) mentioned in the closed-loop analysis with BKS. If Laplace transform is applied to (40) and $\delta(s)$ in (45) is 
substituted into that equation, the closed-loop system with the IBKS can be derived, as follows.

$$
\begin{aligned}
s \mathbf{X}(s) & =\mathbf{A}_{2}(s) \mathbf{X}(s)+\mathbf{B}_{2}(s) \alpha_{c}(s) \\
\mathbf{A}_{2}(s) & =\left[\begin{array}{cc}
a_{2,11}(s) & a_{2,12}(s) \\
a_{2,21}(s) & a_{2,22}(s)
\end{array}\right] \\
& =\left[\begin{array}{cc}
Z_{\alpha}^{*} \\
M_{\alpha}^{*}-\frac{M_{\delta}^{*}}{\phi(s)} \nu_{2, \alpha}(s) & M_{q}^{*}-\frac{M_{\delta}^{*}}{\phi(s)} \nu_{2, q}(s)
\end{array}\right] \\
\mathbf{B}_{2}(s) & =\left[\begin{array}{c}
0 \\
\frac{M_{\delta}^{*}}{\phi(s)}\left(C_{1} C_{2}+1\right)
\end{array}\right] \\
\mathbf{C}(s) & =\left[\begin{array}{ll}
1 & 0
\end{array}\right]
\end{aligned}
$$

Transfer function for the closed-loop system with IBKS can be derived as below.

$$
\begin{aligned}
\frac{\alpha(s)}{\alpha_{c}(s)} & =\mathbf{C}(s)\left(s \mathbf{I}-\mathbf{A}_{2}(s)\right)^{-1} \mathbf{B}_{2}(s) \\
& =\frac{a_{2,12} \frac{M_{\delta}^{*}}{\phi(s)}\left(C_{1} C_{2}+1\right)}{s^{2}-\left(a_{2,11}+a_{2,22}\right) s+\left(a_{2,11} a_{2,22}-a_{2,12} a_{2,21}\right)} \\
& =\frac{T(s)}{s^{2}+2 \zeta \omega_{n} s+\omega_{n}^{2}}
\end{aligned}
$$

where

$$
\begin{aligned}
T(s) & =a_{2,12}(s) \frac{M_{\delta}^{*}}{\phi(s)}\left(C_{1} C_{2}+1\right)=\frac{M_{\delta}^{*}}{\phi(s)}\left(C_{1} C_{2}+1\right) \\
2 \zeta \omega_{n}= & -\left(a_{2,11}(s)+a_{2,22}(s)\right) \\
= & -\left\{Z_{\alpha}^{*}+M_{q}^{*}-\frac{M_{\delta}^{*}}{\phi(s)}\left(C_{1}+C_{2}+M_{q}^{*}+\hat{Z}_{\alpha}^{*}\right)\right\} \\
\omega_{n}^{2}= & a_{2,11}(s) a_{2,22}(s)-a_{2,12}(s) a_{2,21}(s) \\
= & \left(1-\frac{M_{\delta}^{*}}{\phi(s)}\right)\left(Z_{\alpha}^{*} M_{q}^{*}-M_{\alpha}^{*}\right) \\
& +\frac{M_{\delta}}{\phi(s)}\left\{C_{2}\left(\hat{Z}_{\alpha}^{*}-Z_{\alpha}^{*}\right)+\left(C_{1} C_{2}+1\right)\right\}
\end{aligned}
$$

When the uncertainties doesn't exist in any aerodynamic derivative estimates, (47) becomes identical to (19), which is the closed-loop transfer function with IBKS for the nominal case.

\section{APPENDIX C}

AERodynamic DeRIVATIVES FOR Simulation

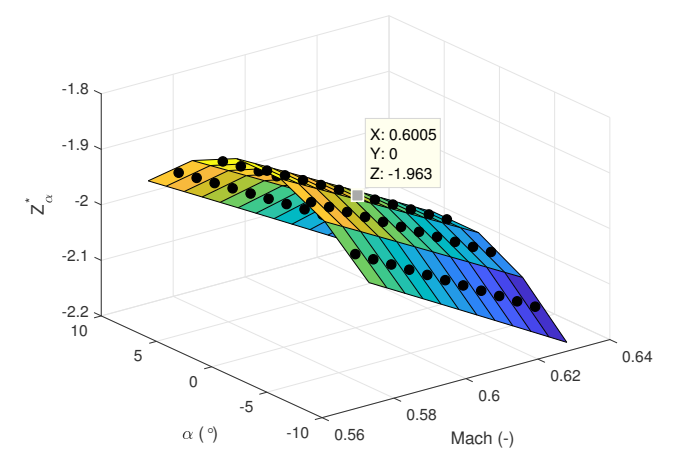

(a) $Z_{\alpha}^{*}$

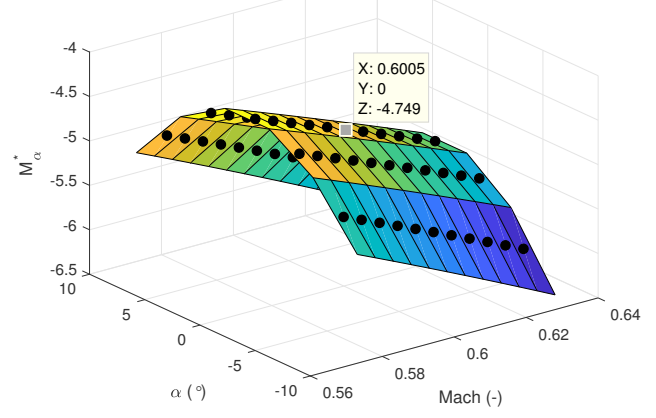

(b) $M_{\alpha}^{*}$

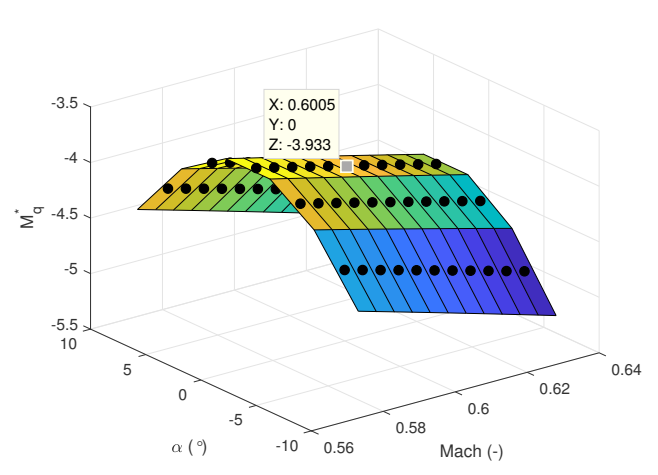

(c) $M_{q}^{*}$

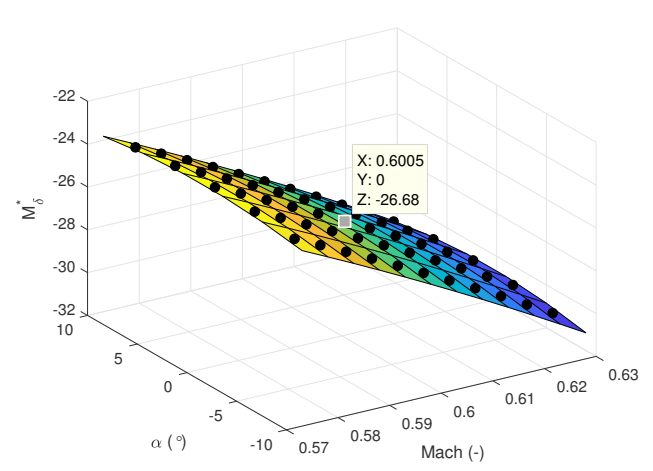

(d) $M_{\delta}^{*}$

Fig. 7. Aerodynamic Derivatives for Simulation

\section{ACKNOWLEDGMENT}

This research is co-funded by the European Union in the scope of INCEPTION project, which has received funding from the EUs Horizon2020 Research and Innovation Programme under grant agreement No. 723515.

\section{REFERENCES}

[1] P. V. Kokotovic, "The joy of feedback: nonlinear and adaptive," IEEE Control Systems Magazine, vol. 12, no. 3, pp. 7-17, 1992.

[2] T. Lee and Y. Kim, "Nonlinear adaptive flight control using backstepping and neural networks controller," Journal of Guidance, Control, and Dynamics, vol. 24, no. 4, pp. 675-682, 2001.

[3] J. Farrell, M. Sharma, and M. Polycarpou, "Backstepping-based flight control with adaptive function approximation," Journal of Guidance, Control, and Dynamics, vol. 28, no. 6, pp. 1089-1102, 2005. 
[4] H.-S. Ju and C.-C. Tsai, "Longitudinal axis flight control law design by adaptive backstepping," IEEE Transactions on Aerospace and Electronic Systems, vol. 43, no. 1, pp. 311-329, 2007.

[5] L. Sun and W. Huo, "6-dof integrated adaptive backstepping control for spacecraft proximity operations," IEEE Transactions on Aerospace and Electronic Systems, vol. 51, no. 3, pp. 2433-2443, 2015.

[6] L. G. Carrillo, A. Dzul, and R. Lozano, "Hovering quad-rotor control: A comparison of nonlinear controllers using visual feedback," IEEE Transactions on Aerospace and Electronic Systems, vol. 48, no. 4, pp. 3159-3170, 2012.

[7] X. Huang and Y. Yan, "Saturated backstepping control of underactuated spacecraft hovering for formation flights," IEEE Transactions on Aerospace and Electronic Systems, vol. 53, no. 4, pp. 1988-2000, 2017.

[8] B. Lian and H. Bang, "Momentum transfer-based attitude control of spacecraft with backstepping," IEEE transactions on aerospace and electronic systems, vol. 42, no. 2, pp. 453-463, 2006.

[9] Y. Kim and B. S. Kim, "Pitch autopilot design for agile missiles with uncertain aerodynamic coefficients," IEEE Transactions on Aerospace and Electronic Systems, vol. 49, no. 2, pp. 907-914, 2013.

[10] J. Ghommam and M. Saad, "Autonomous landing of a quadrotor on a moving platform," IEEE Transactions on Aerospace and Electronic Systems, vol. 53, no. 3, pp. 1504-1519, 2017.

[11] X. Huang and Y. Yan, "Saturated backstepping control of underactuated spacecraft hovering for formation flights," IEEE Transactions on Aerospace and Electronic Systems, vol. 53, no. 4, pp. 1988-2000, 2017.

[12] P. van Gils, E.-J. Van Kampen, C. C. de Visser, and Q. P. Chu, "Adaptive incremental backstepping flight control for a high-performance aircraft with uncertainties," in AIAA Guidance, Navigation, and Control Conference, 2016.

[13] A. Ait Haddou Ali, Q. P. Chu, E.-J. Van Kampen, and C. C. de Visser, "Exploring adaptive incremental backstepping using immersion and invariance for an f-16 aircraft," in AIAA Guidance, Navigation, and Control Conference, 2014.

[14] P. Acquatella, E. van Kampen, and Q. P. Chu, "Incremental backstepping for robust nonlinear flight control," in Proceedings of the EuroGNC 2013, 2nd CEAS Specialist Conference on Guidance, Navigation and Control, 2013, pp. 1444-1463.

[15] G. P. Falconí, V. A. Marvakov, and F. Holzapfel, "Fault tolerant control for a hexarotor system using incremental backstepping," in 2016 IEEE Conference on Control Applications (CCA). IEEE, 2016, pp. 237-242.

[16] S. Sieberling, Q. Chu, and J. Mulder, "Robust flight control using incremental nonlinear dynamic inversion and angular acceleration prediction," Journal of guidance, control, and dynamics, vol. 33, no. 6, pp. 17321742, 2010.

[17] E. J. Smeur, Q. Chu, and G. C. de Croon, "Adaptive incremental nonlinear dynamic inversion for attitude control of micro air vehicles," Journal of Guidance, Control, and Dynamics, vol. 38, no. 12, pp. 450461, 2015

[18] X. Wang, E.-J. Van Kampen, Q. Chu, and P. Lu, "Stability analysis for incremental nonlinear dynamic inversion control," Journal of Guidance, Control, and Dynamics, vol. 42, no. 5, pp. 1116-1129, 2019.

[19] P. Acquatella, W. Falkena, E.-J. van Kampen, and Q. P. Chu, "Robust nonlinear spacecraft attitude control using incremental nonlinear dynamic inversion." in AIAA Guidance, Navigation, and Control Conference, 2012.

[20] P. Simplício, M. Pavel, E. Van Kampen, and Q. Chu, "An acceleration measurements-based approach for helicopter nonlinear flight control using incremental nonlinear dynamic inversion," Control Engineering Practice, vol. 21, no. 8, pp. 1065-1077, 2013.

[21] S. H. Lane and R. F. Stengel, "Flight control design using non-linear inverse dynamics," Automatica, vol. 24, no. 4, pp. 471-483, 1988.

[22] C.-H. Lee, B.-E. Jun, and J.-I. Lee, "Connections between linear and nonlinear missile autopilots via three-loop topology," Journal of Guidance, Control, and Dynamics, pp. 1426-1432, 2016.

[23] Y. Xu, "Multi-timescale nonlinear robust control for a miniature helicopter," IEEE Transactions on Aerospace and Electronic systems, vol. 46, no. 2, pp. 656-671, 2010.

[24] R. Lungu, M. Lungu, and L. T. Grigorie, "Automatic control of aircraft in longitudinal plane during landing," IEEE Transactions on Aerospace and Electronic Systems, vol. 49, no. 2, pp. 1338-1350, 2013.

[25] Y. Li, Z. Jing, and G. Liu, "Maneuver-aided active satellite tracking using six-dof optimal dynamic inversion control," IEEE Transactions on Aerospace and Electronic Systems, vol. 50, no. 1, pp. 704-719, 2014.

[26] D. McLean, Automatic flight control systems. Prentice Hall, New York, 1990.

[27] F. Golnaraghi and B. C. Kuo, Automatic Control Systems. John Wiley and Sons Ltd, 2009.
[28] L. Sonneveldt, Q. Chu, and J. Mulder, "Nonlinear flight control design using constrained adaptive backstepping," Journal of Guidance, Control, and Dynamics, vol. 30, no. 2, pp. 322-336, 2007.

[29] H. K. Khalil, Nonlinear systems. Upper Saddle River, 1996.

[30] J.-J. E. Slotine, W. Li et al., Applied nonlinear control. Prentice hall Englewood Cliffs, NJ, 1991.

[31] I. K. M. Krstic and P. Kokotovic, Nonlinear and Adaptive Control. John Wiley and Sons, Inc., New York, 1995.

[32] B. K. et al., Flight Dynamics and Control. Kyung Moon Sa, 2004.

[33] B.-J. Jeon, M.-G. Seo, H.-S. Shin, and A. Tsourdos, "Understandings of the incremental backstepping control through theoretical analysis under the model uncertainties," in 2018 IEEE Conference on Control Technology and Applications (CCTA). IEEE, 2018, pp. 318-323.

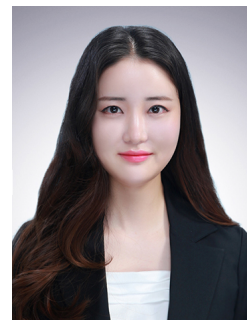

Byoung-Ju Jeon Byoung-Ju Jeon received the B.S. and M.S. degrees in aerospace engineering from Korea Advanced Institute of Science and Technology (KAIST), Daejeon, Korea, in 2012 and 2014, respectively. She is currently a Ph.D. candidate at Cranfield University. Her current research interests include automatic control and intelligent systems.

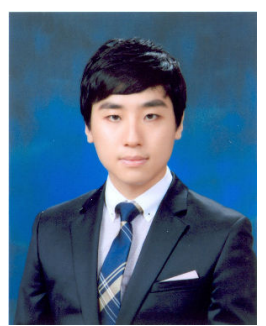

Min-Guk Seo Min-Guk Seo received the B.S., M.S. and $\mathrm{Ph} . \mathrm{D}$. degrees in aerospace engineering from Korea Advanced Institute of Science and Technology (KAIST), Daejeon, Korea, in 2012, 2014 and 2017, respectively. $\mathrm{He}$ is currently a research fellow in autonomous systems at Cranfield University. His current research interests include guidance, navigation and control of autonomous systems.

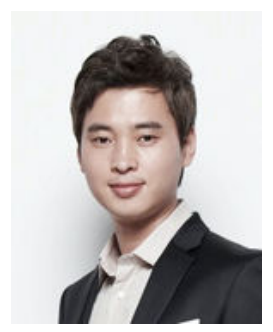

Hyo-Sang Shin received his BSc from Pusan National University in 2004 and gained an MSc on flight dynamics, guidance and control in Aerospace Engineering from KAIST and a PhD on cooperative missile guidance from Cranfield University in 2006 and 2010, respectively. He is currently Professor on Guidance, Control and Navigation Systems in Autonomous and Intelligent Systems Group at Cranfield University. His current research interests include multiple target tracking, probabilistic target detection and distributed control of multiple agent

systems.

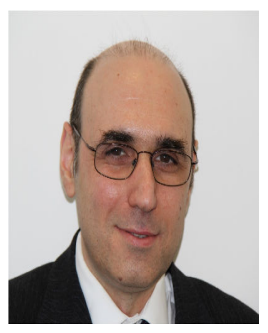

Antonios Tsourdos obtained a MEng in electronic, control and systems engineering from the University of Sheffield (1995), an MSc in systems engineering from Cardiff University (1996), and a $\mathrm{PhD}$ in nonlinear robust missile autopilot design and analysis from Cranfield University (1999). He is a Professor of Control Engineering with Cranfield University, and was appointed Head of the Centre for CyberPhysical Systems in 2013. He was a member of the Team Stellar, the winning team for the UK MoD Grand Challenge (2008) and the IET Innovation Award (Category Team, 2009). 
$2019-11-11$

Understandings of classical and incremental backstepping controllers with model uncertainties

Jeon, Byoung-Ju

IEEE

Jeon B-J, Seo M-G, Shin H-S, Tsourdos A. (2020) Understandings of classical and incremental backstepping controllers with model uncertainties. IEEE Transactions on Aerospace and Electronic Systems, Volume 56, Issue 4, August 2020, pp. 2628-2641

https://doi.org/10.1109/TAES.2019.2952631

Downloaded from Cranfield Library Services E-Repository 